BSG 2014 abstracts

\begin{tabular}{|c|c|c|c|c|c|c|c|c|c|}
\hline \multirow[b]{2}{*}{ Centre } & \multirow[b]{2}{*}{ First list } & \multirow{2}{*}{$\begin{array}{l}\text { Endoscopists } \\
\text { (BCSP colonoscopists) }\end{array}$} & \multicolumn{4}{|c|}{ Endoscopist grade } & \multirow[b]{2}{*}{ Lists per week } & \multirow[b]{2}{*}{ Evenings/ Weekends } & \multirow[b]{2}{*}{ SSPs per list } \\
\hline & & & Consultant & Registrar & Staff/ other grade & Nurse endoscopist & & & \\
\hline Norwich & 08.07 .13 & $6(1)$ & 0 & 3 & 0 & 3 & 6 & Yes/Yes & $1^{*}$ \\
\hline South of Tyne & 07.05 .13 & $2(0)$ & 0 & 1 & 1 & 0 & 2.5 & Yes/Yes & 2 \\
\hline St Marks & 22.07 .13 & $8(2)$ & 1 & 5 & 0 & 2 & 7 & Yes/No & $1^{*}$ \\
\hline Surrey & 07.11 .13 & $6(6)$ & 6 & 0 & 0 & 0 & 2 & No/Yes & 2 \\
\hline West Kent and Medway & 11.06 .13 & 7 (3) & 1 & 0 & 5 & 1 & 4 & No/Yes & 2 \\
\hline W'hampton & 07.08 .13 & $6(3)$ & 4 & 0 & 2 & 0 & 6 & No/No & 1 \\
\hline
\end{tabular}

Introduction The NHS Bowel Cancer Screening Programme (BCSP) is being expanded to include a single flexible sigmoidoscopy (FSIG) called BowelScope, offered to all 55 year olds in addition to biannual faecal occult blood testing from age 60-75 years.

6 pilot sites began BowelScope screening in May 2013, with a view to full English coverage by the end of 2016 .

Methods We aim to describe practical issues involved in the delivery of BowelScope screening at the pilot centres, covering unit set-up, list format, and endoscopists delivering lists.

A survey was sent to the 6 pilots for completion by screening staff. Data were also retrieved from the national BCSP database.

Results The first BowelScope list was delivered in the South of Tyne Screening Centre on $7^{\text {th }}$ May 2013. By December 2013, 4135 flexible sigmoidoscopy procedures had been performed in 6 centres.

Centres have delivered 20-80 lists each, performing 2-7 lists per week. Sessions are run at varying times of day including evenings and Saturdays.

35 endoscopists undertake lists regularly, of whom 15 were already BCSP accredited. Other BCSP colonoscopists provide back up for lists when required. All non-BCSP were accredited through a combination of direct observation of procedural skills (DOPS) and an MQC exam.

Specialist Screening Practitioners (SSPs) attend all lists, and are deployed in a variety of ways including: following patient journey, consenting or giving information, and supervision assistant SSPs.

Table 1 shows details of screening lists by centre.

Conclusion BowelScope screening is being successfully delivered at the six pilot centres. Each centre has developed a screening template and organisational pattern that works around patient needs and existing endoscopy and bowel screening services. New patterns of working have been required to deliver BowelScope and challenges remain regarding adequate numbers of endoscopists.

Disclosure of Interest None Declared.

\section{PWE-057 A COMPOSITE MEASURE OF COLONIC INTUBATION (CIRC) IS BETTER ABLE TO DISTINGUISH PERFORMANCE OF COLONOSCOPY AND IS ASSOCIATED WITH HIGHER POLYP DETECTION RATES}

${ }^{1} \mathrm{R}$ Valori ${ }^{*},{ }^{2} S$ Damery, ${ }^{1} E$ Swarbrick, ${ }^{1} G$ Williams, ${ }^{1} \mathrm{~J}$ Anderson, ${ }^{1} \mathrm{M}$ Donnelly, ${ }^{1} \mathrm{D}$ Gavin. ${ }^{1} B S G / A C P G B I$, National Colonoscopy Audit Group; 'Department of Primary Care, Birmingham University, Birmingham, UK

10.1136/gutjnl-2014-307263.317

Introduction Caecal intubation rate (CIR) is a key performance indicator of colonoscopy. CIR with acceptable tolerance is only achieved in some patients with more sedation. As most colonoscopists achieve $>90 \%$ intubation, CIR alone cannot define excellence. This study proposes a more discerning, composite measure of colonoscopy intubation $\left(\mathrm{CIR}^{\mathrm{C}}\right)$ combining $\mathrm{CIR}$, patient comfort and sedation. Characteristics of patient, unit, equipment and colonoscopist associated with $\mathrm{CIR}^{\mathrm{C}}$, and the relation of $\mathrm{CIR}^{\mathrm{C}}$ to pathology detection are reported.

Methods Data from 20085 colonoscopies reported in the 2011 UK national audit (Gut 2013;62:242-249) were used for this analysis. The $\mathrm{CIR}^{\mathrm{C}}$ was defined as percentage of procedures achieving caecal intubation with median dose of midazolam or less, and nurse assessed comfort score of 1-3 (max 5). Multivariate analysis using binary logistic regression assessed patient, unit and colonoscopist factors in order to derive Odds Ratios (ORs) and $95 \%$ confidence intervals (CIs) for factors independently associated with $\mathrm{CIR}^{\mathrm{c}}$ after controlling for effects of all model variables. ORs have a significance level of $<0.001$ unless stated.

Results Overall achievement of $\mathrm{CIR}^{\mathrm{C}}$ was $54.1 \%$ ( $\mathrm{n}=10865$ procedures). The $\mathrm{CIR}^{\mathrm{C}}$ was better able to distinguish differences in performance than single measures such as CIR and polyp detection. Older age, male sex (OR 1.40; CI:1.32-1.49), adequate or excellent bowel prep and FOBT screen positive as indication were all associated with a higher chance of achieving $\mathrm{CIR}^{\mathrm{C}}$. Unit (JAG) accreditation (OR 1.26; CI 1.16-1.35) and the presence of one or more magnetic imagers in the unit (OR 1.29; 1.19-1.40) were associated with higher CIR $^{\mathrm{C}}$. Surgical speciality, greater annual volume, fewer years' experience and higher training/trainer status were associated with higher $\mathrm{CIR}^{\mathrm{C}}$. Achieving the $\mathrm{CIR}^{\mathrm{C}}$ was associated with significantly higher polyp detection rates (OR 1.12; 1.04-1.20) and higher cancer detection rates (OR 1.14; 0.98-1.32, $\mathrm{p}=0.10$ ).

Conclusion The $\mathrm{CIR}^{\mathrm{C}}$ provides a richer picture of colonoscopic intubation than CIR alone and is better able to discern factors associated with intubation competence in relation to patients, units, equipment and colonoscopists. Unit (JAG) accreditation was associated with higher $\mathrm{CIR}^{\mathrm{C}}$. Colonoscopists who perform more procedures, who have more experience of training or being trained, who have practised fewer years and who are in a surgical speciality had significantly higher rates of $\mathrm{CIR}^{\mathrm{C}}$. The $\mathrm{CIR}^{\mathrm{C}}$ was associated with a significantly higher polyp detection rate. It is proposed that $\mathrm{CIR}^{\mathrm{C}}$ replaces CIR as the key performance indicator for intubation of the colon.

Disclosure of Interest None Declared.

\section{PWE-058 INCREASING USE OF ENDOSCOPIC RESECTION, SINGLE-CENTRE EXPERIENCE}

S Ansari, R Sood*, V Hegade, L-A Smith. Digestive Diseases Centre, Bradford Royal Infirmary, Bradford, UK

\subsection{6/gutjnl-2014-307263.318}

Introduction Endoscopic Resection (ER), a technique, first pioneered in Japan for treating early gastric cancers, has gained 